\title{
Spectral editing through laser-flash excitation in two-dimensional photo-CIDNP MAS NMR experiments
}

\author{
Karthick Babu Sai Sankar Gupta ${ }^{a}$, Eugenio Daviso ${ }^{\mathrm{a}, \mathrm{b}}$, Gunnar Jeschke ${ }^{\mathrm{c}}$, A. Alia ${ }^{\mathrm{a}, \mathrm{d}}$, Matthias Ernst ${ }^{\mathrm{c}}$, \\ Jörg Matysik ${ }^{\mathrm{a}, \mathrm{d}, *}$ \\ ${ }^{a}$ Leiden Institute of Chemistry, Leiden University, P.O. Box 9502, 2300 RA Leiden, The Netherlands \\ ${ }^{\mathrm{b}}$ Francis Bitter Magnet Laboratory, Albany Street 150, NW14, Cambridge, MA 02139, USA \\ 'ETH Zürich, Physical Chemistry, Wolfgang-Pauli-Strasse 10, 8093 Zürich, Switzerland \\ d Institut für Analytische Chemie, Universität Leipzig, Linnéstr. 3, 04103 Leipzig, Germany
}

\section{A R T I C L E I N F O}

\section{Article history:}

Received 29 April 2014

Revised 8 June 2014

Available online 27 June 2014

\section{Keywords:}

Photo-CIDNP

MAS NMR

Spectral editing

Hyperfine interaction

laser excitation

DARR

\begin{abstract}
A B S T R A C T
In solid-state photochemically induced dynamic nuclear polarization (photo-CIDNP) MAS NMR experiments, strong signal enhancement is observed from molecules forming a spin-correlated radical pair in a rigid matrix. Two-dimensional ${ }^{13} \mathrm{C}-{ }^{13} \mathrm{C}$ dipolar-assisted rotational resonance (DARR) photo-CIDNP MAS NMR experiments have been applied to obtain exact chemical shift assignments from those cofactors. Under continuous illumination, the signals are enhanced via three-spin mixing (TSM) and differential decay (DD) and their intensity corresponds to the electron spin density in $\mathrm{p}_{\mathrm{z}}$ orbitals. In multiple- ${ }^{13} \mathrm{C}$ labelled samples, spin diffusion leads to propagation of signal enhancement to all ${ }^{13} \mathrm{C}$ spins. Under steady-state conditions, direct signal assignment is possible due to the uniform signal intensity. The original intensities, however, are inaccessible and the information of the local electron spin density is lost. Upon laser-flash illumination, the signal is enhanced via the classical radical pair mechanism (RPM). The obtained intensities are related to isotropic hyperfine interactions $a_{\text {iso }}$ and both enhanced absorptive and emissive lines can be observed due to differences in the sign of the local isotropic hyperfine interaction. Exploiting the mechanism of the polarization, selectivity can be increased by the novel time-resolved two-dimensional dipolar-assisted rotational resonance (DARR) MAS NMR experiment which simplifies the signal assignment compared to complex spectra of the same RCs obtained by continuous illumination. Here we present two-dimensional time-resolved photo-CIDNP MAS NMR experiments providing both directly: signal assignment and spectral editing by sign and strength of $a_{\text {iso }}$. Hence, this experiment provides a direct key to the electronic structure of the correlated radical pair.
\end{abstract}

(c) 2014 Elsevier Inc. All rights reserved.

\section{Introduction}

The discovery of the solid-state photo-CIDNP (photochemically induced dynamic nuclear polarization) effect (for reviews: [1,2]) by Zysmilich and McDermott in 1994 in frozen and quinone-blocked bacterial reaction centers (RCs) of Rhodobacter (R.) sphaeroides R26 by ${ }^{15} \mathrm{~N}$ magic-angle spinning (MAS) NMR under continuous illumination with white light offered a new experimental access to electron-nuclear processes during charge separation [3]. By induction of a non-Boltzmann nuclear spin polarization, enhancement of signals of a factor of more than 80,000 has been observed from the nuclei of the cofactors forming the light-induced radical

\footnotetext{
* Corresponding author at: Institut für Analytische Chemie, Universität Leipzig Linnéstr. 3, 04103 Leipzig, Germany. Fax: +49 3419736115.

E-mail address: joerg.matysik@uni-leipzig.de (J. Matysik).
}

pair [4-7]. In recent years, the solid-state photo-CIDNP effect has also been observed in several photosynthetic RCs, as those obtained from the purple bacteria $R$. sphaeroides wildtype (WT) [4] and R26 [3,5,7], Rhodopseudomonas acidophila [8], the green sulfur bacterium Chlorobium tepidum [9], the heliobacterium Heliobacillus mobilis [6] as well as of the photosystems I and II of plants [10-12] and algae [13]. The occurrence of the solid-state photoCIDNP effect appears to be an intrinsic property of natural photosynthetic RCs [14]. Recent observation of the effect in a blue-light photoreceptor [15] demonstrates that the effect is not limited to natural photosynthesis.

In RCs of $R$. sphaeroides, light absorption induces charge separation within 3 ps from the primary donor $(\mathrm{P})$ formed by 2 bacteriochlorophyll $a$ (BChl) to the primary acceptor, a bacteriopheophytin $\left(\Phi_{\mathrm{A}}\right)$ (for review, see [16]) (Fig. 1A and B). The radical pair is initially in a singlet state (Fig. 2). From this singlet state, 

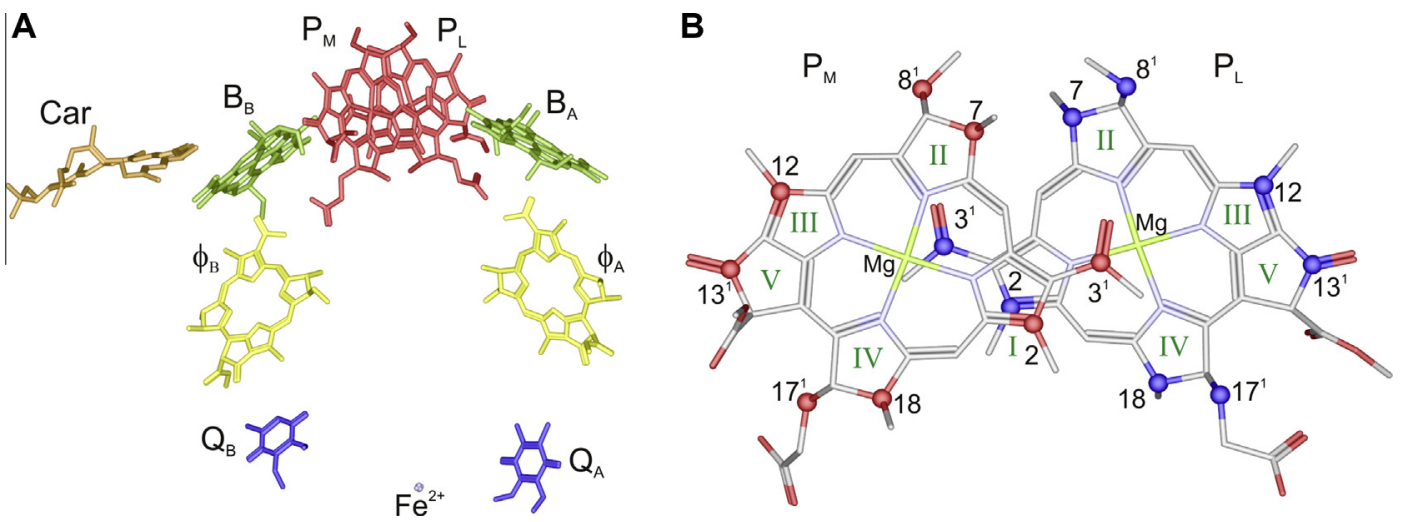

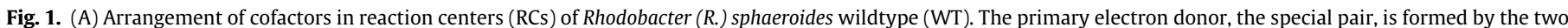

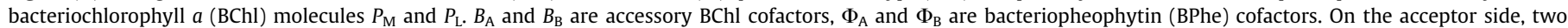

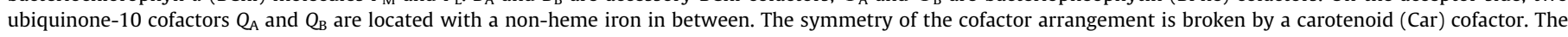

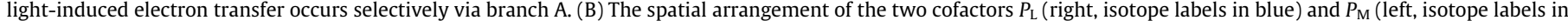

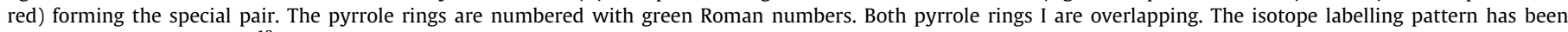

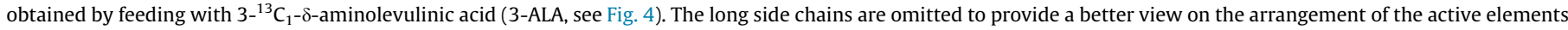

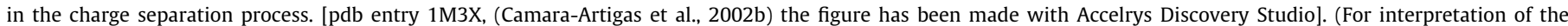
references to color in this figure legend, the reader is referred to the web version of this article.)

photochemically induced dynamic electron polarization (photoCIDEP) is observed as strongly enhanced absorptive and emissive signals in the EPR spectrum $[17,18]$. During the lifetime of the radical pair, the electron-spin system oscillates between the singlet state $(S)\left[{ }^{1}\left(\mathrm{P}^{+} \Phi_{\mathrm{A}}{ }^{-}\right)\right]$of the radical pair and the triplet state $\left(T_{0}\right)$ $\left[{ }^{3}\left(\mathrm{P}^{+} \Phi_{\mathrm{A}}^{-}\right)\right]$with a frequency that depends on both the hyperfine interaction and the difference of the electron Zeeman interaction.

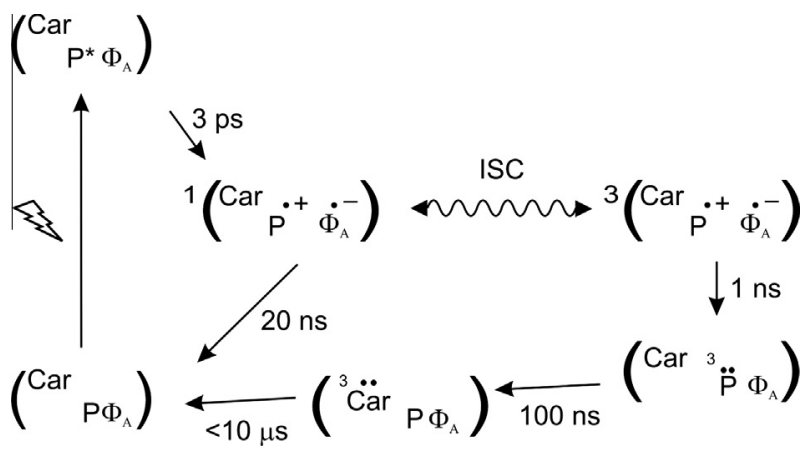

Fig. 2. Kinetics and spin dynamics of electron transport in quinone-depleted RCs of $R$. sphaeroides wild type (WT). After absorption of a photon the photochemically excited state of the primary donor $P^{*}$ is formed and an electron is transferred to the primary acceptor $\Phi_{\mathrm{A}}$, a bacteriopheophytin cofactor. Initially, the radical pair is in its singlet state ${ }^{1}\left(\mathrm{P}^{+}+\Phi_{\mathrm{A}}{ }^{-}\right)$. It evolves into a triplet state ${ }^{3}\left(\mathrm{P}^{+} \Phi_{\mathrm{A}}^{-}\right)$due to the electronic interactions and hyperfine coupling with nearby nuclei, a process which is known as intersystem crossing (ISC). The radical-pair mechanism (RPM) leads to sorting of nuclear spins via the isotropic hyperfine coupling, but without a net increase of the population difference of the spin up and the spin down nuclear states. In the TSM, hyperfine coupling, nuclear and electronic Zeeman interactions, and anisotropic interactions involving electrons and nuclei lead to symmetry breaking and net nuclear polarization that can be observed both in laser excitation experiments and under steady-state conditions. The lifetime for recombination from the singlet state to the ground state is $20 \mathrm{~ns}$, while charge recombination from the ${ }^{3}\left(\mathrm{P}^{+} \Phi_{\mathrm{A}}{ }^{-}\right)$radical pair state forms a donor triplet state ${ }^{3} \mathrm{P}$ with a time constant of $1 \mathrm{~ns}$. With continuous illumination the difference in recombination rates from the singlet and triplet states to the neutral ground state also break the symmetry when they match the inverse of the pseudosecular component of the hyperfine interaction, a process that is known as the DD mechanism. For the WT, the ${ }^{3} \mathrm{P}$ is rapidly converted $(100 \mathrm{~ns})$ in a carotenoid triplet $\left({ }^{3} \mathrm{Car}\right)$ and followed by a much slower decay from the carotenoid triplet state to the ground state. Time-resolved experiments have shown that a large fraction of the excited state decays via ${ }^{3} \mathrm{Car}$, in competition with back conversion to the ${ }^{3}\left(\mathrm{P}^{+} \Phi_{\mathrm{A}}^{-}\right)$that decays rapidly with $20 \mathrm{~ns}$ to establish the steady state. Before reaching this steady state, however, transient effects from RPM, TSM and DD can be observed with time-resolved photo-CIDNP, on a timescale of $10 \mu$ s and different mechanisms can be resolved by adjusting the time between the photo-CIDNP excitation and the NMR detection scheme.
The coherent interconversion from singlet to triplet radical pairs and back gives rise to spin sorting within the scheme of the well-known radical pair mechanism (RPM) (Fig. 2) [19,20]. Under continuous illumination (CI), the RPM does not induce signal enhancement because the reaction products of both decay branches are have opposite sign and cancel exactly.

In solid-state photo-CIDNP MAS NMR experiments under CI, two additional solid-state mechanisms run in parallel to induce net nuclear polarization which remains under steady-state conditions (Fig. 2) [1,2]: (i) Electron-electron-nuclear three-spin mixing (TSM) breaks the balance by coherent evolution of the correlated radical pair state in interaction with the nuclear spins and the applied magnetic field, depending on the signs of the electronelectron and of the anisotropic electron-nuclear interactions $[21,22]$. (ii) In the electron-nuclear differential decay (DD) mechanism [23], the symmetry is broken by different lifetimes of the $S$ $\left[{ }^{1}\left(\mathrm{P}^{+} \Phi_{\mathrm{A}}{ }^{-}\right)\right]$and of the $T_{0}\left[{ }^{3}\left(\mathrm{P}^{+} \Phi_{\mathrm{A}}^{-}\right)\right]$states. The dependence of secular part of the hyperfine coupling $A$ is the only single matching of interactions $2\left|\omega_{\mathrm{I}}\right|=|A|$ is required and the difference of singlet and triplet radical pair lifetimes must be of the order of the inverse hyperfine coupling [1]. A more detailed analysis shows [24] that ${ }^{13} \mathrm{C}$ the hyperfine coupling anisotropy $\Delta A$ required for these solid-state mechanisms scales with the local electron spin density in the $\mathrm{p}_{\mathrm{z}}$ orbitals of the $\pi$ system of the radical cation state. PhotoCIDNP intensities in this regime are roughly proportional to $\Delta A^{2}$ [1].

In time-resolved experiments on RCs of $R$. sphaeroides WT, transient nuclear polarization has been observed long as $10 \mu \mathrm{s}$ [25]. The decay from the singlet state to the ground state is $20 \mathrm{~ns}$, much faster than the pathway via the donor triplet state ${ }^{3} \mathrm{P}$, which is rate limited by the decay from the carotenoid triplet state to the ground state, i.e., $10 \mu$ s (Fig. 1). Thus, transient nuclear polarization can be observed, originating from the nuclear polarization associated with rapid decay of the ${ }^{1}\left(\mathrm{P}^{++} \Phi_{\mathrm{A}}^{--}\right)$state while the polarization associated with the decay of the ${ }^{3} \mathrm{P}$ channel is hidden by the paramagnetic triplet state of the carotenoid $\left({ }^{3} \mathrm{Car}\right)$ during its lifetime. As a result, the nuclear polarization of the triplet decay channel cannot be detected on the nearby nuclei in experiments with a short delay $<10 \mu$ s between optical excitation and NMR detection (Fig. 2) $[2,25]$. Hence, transiently, using time-resolved experiments, RPM nuclear polarization can be observed under solid-state conditions since the triplet decay rate differs from the singlet one [25]. The sign of the transient light-induced signals follows Kaptein's sign 
rules [26]. According to those rules, in the RPM the sign depends on the spin state of the precursor, the decay path of the radical pair, and the signs of $\Delta g$ of the radical pair and of the hyperfine (hf) constant. If, as in our case, the first three parameters are given by the system, the sign and signal intensity are directly correlated to the hf constant $a_{\text {iso. }}$.

Hence, time-resolved photo-CIDNP MAS NMR nuclear intensities allows to map isotropic electron spin densities and corresponding hf interaction constants $\left(a_{\text {iso }}\right)$ on the donor with atomic selectivity [27]. Interpretation of the one-dimensional envelope of transient nuclear polarization requires chemical shift assignments, which have been obtained in separate continuous-illumination experiments. Here we show that two-dimensional nanosecond laser-flash photo-CIDNP MAS NMR experiments measure both, chemical shifts, that are unambiguously assigned, and local electron spin densities in a single experiment.

\section{Methodology}

\subsection{Dipolar assisted rotational resonance (DARR) spectroscopy}

To explore connectivities between carbons, ${ }^{13} \mathrm{C}-{ }^{13} \mathrm{C}$ homonuclear shift-correlation experiments are available. In solid state MAS NMR, several pulse sequences have been developed for homonuclear correlations, such as DRAMA [28], RFDR [29,30], C7 [31], PDSD [32,33] and DARR [34]. More on dipolar recoupling could found on these review articles [35,36]. In the DARR (Dipolar assisted rotational resonance) experiment, nuclear polarization transfer is driven by a spin-diffusion-type mechanism while the heteronuclear $\left({ }^{1} \mathrm{H}-{ }^{13} \mathrm{C}\right)$ dipolar couplings are re-established by continuous ${ }^{1} \mathrm{H}$ rf irradiation on a rotary resonance condition [37]. The pulse sequence for the DARR experiment is shown in Fig. 3A. In the preparation period, a standard cross polarization (CP) step

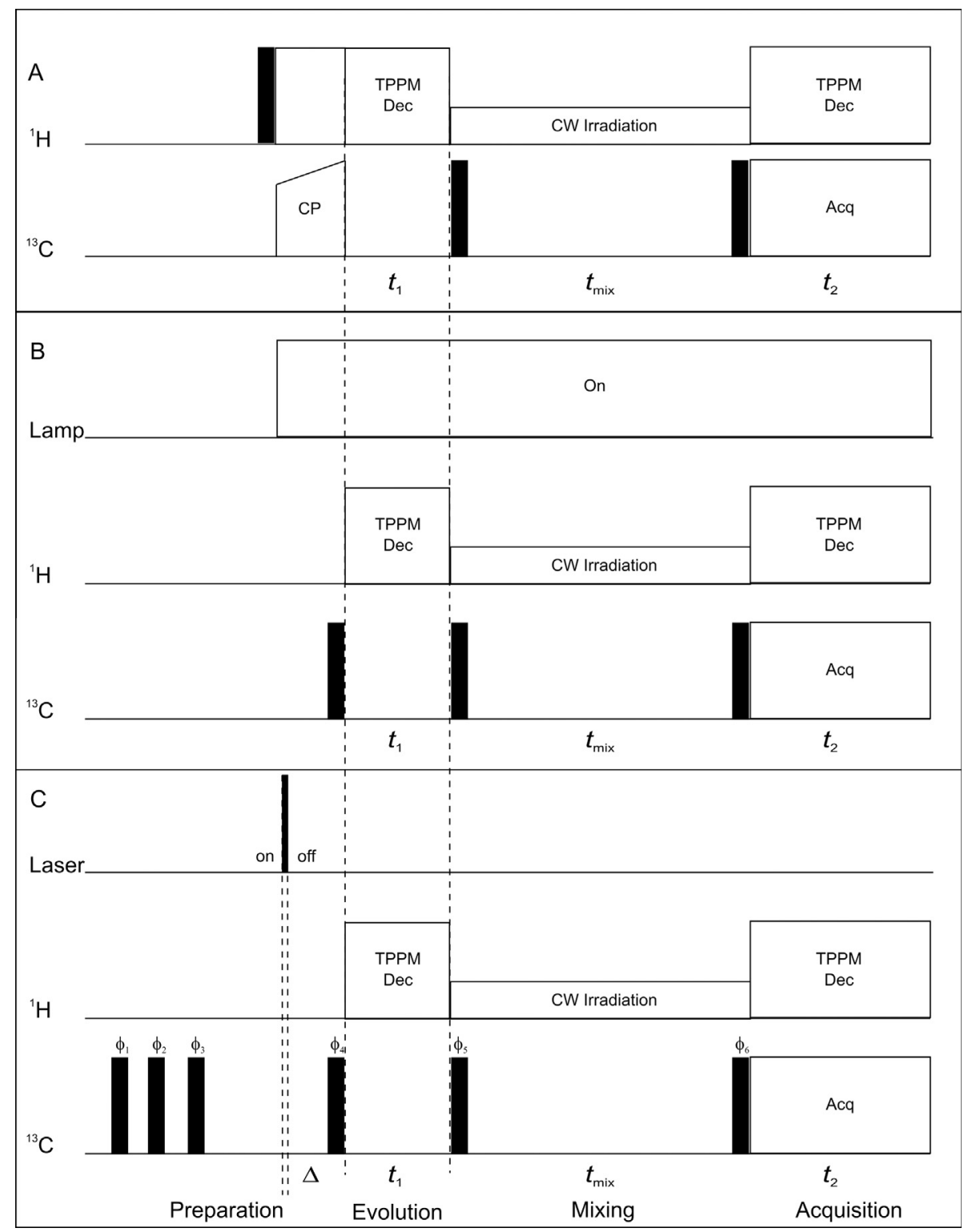

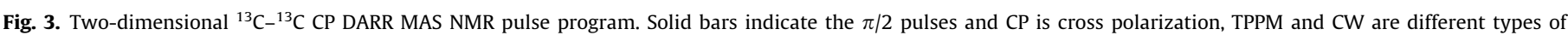

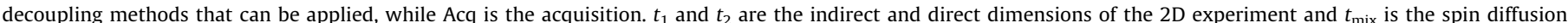

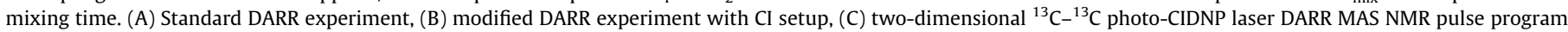

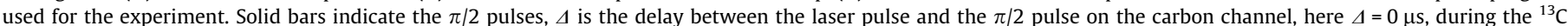

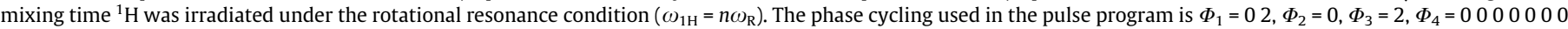
$022222222, \Phi_{5}=00002222, \Phi_{6}=0213$ and receiver $\Phi_{R}=0213203120310213$. Here definitions are: $0=+X, 1=+Y, 2=-X, 3=-Y$. 
is used mainly to enhance the ${ }^{13} \mathrm{C}$ polarization, by factor of $\sim 4$ by transfer of magnetization from the protons. During the evolution period $\left(t_{1}\right)$ and acquisition, $90 \mathrm{kHz}$ TPPM ${ }^{1} \mathrm{H}$ decoupling was used (total phase difference, $15^{\circ}$, TPPM pulse length $5.5 \mathrm{~ms}$ [38]. After the evolution period, the magnetization is tilted along the $z$-axis with a $\pi / 2$ pulse and mixing $\left(t_{\text {mix }}\right)$ occurs longitudinally with low power irradiation on ${ }^{1} \mathrm{H}$. The ${ }^{1} \mathrm{H}$ rf field strength is set to the $n=1$ rotary resonance condition [39].

\subsection{Modified DARR for photo-CIDNP under continuous illumination}

The modified pulse program using the strong initial ${ }^{13} \mathrm{C}$ polarization provided by the solid-state photo-CIDNP effect is displayed in Fig. 3B. The preparation period has been modified from the standard DARR experiment (Fig. 3A) by replacing the CP segment with a simple ${ }^{13} \mathrm{C} \pi / 2$ pulse. Continuous white light is obtained from a $1000 \mathrm{~W}$ xenon arc lamp [40].

\subsection{Modified DARR for photo-CIDNP under laser-flash illumination}

The modified pulse program using the strong initial ${ }^{13} \mathrm{C}$ polarization provided by the solid-state photo-CIDNP effect is displayed in Fig. 3C. The preparation period has been adapted from the standard DARR experiment (Fig. 3A) by removing the CP segment. Advanced photo-CIDNP techniques are used here for selective excitation of the source for the nuclear polarization, and the laser-flash photo-CIDNP segment [41] is incorporated, using a laser light pulse and a subsequent $\pi / 2$ pulse. The presaturation of ${ }^{13} \mathrm{C}$ pulses allows for accelerating the experiment by destroying any residual polarization from the previous $\pi / 2$ pulse [41].

\section{Materials and methods}

\subsection{Sample preparation}

The selective isotopic labelling in RCs of $R$. sphaeroides is achieved by feeding of selectively labelled $3-{ }^{13} C_{1}-\delta$-aminolevulinic acid (3-ALA), which is a precursor for the formation of $\mathrm{BChl}$ and BPhe (Fig. 4), and leads to a ${ }^{13} \mathrm{C}$ enrichment of $\sim 60 \%$ [42]. 3-ALA has been purchased from Buchem B.V. (Apeldoorn, The Netherlands).

The RCs were isolated as described earlier [43] and the quinones were removed by incubating the RCs at a concentration of $0.6 \mu \mathrm{M}$ in 4\% LDAO, $10 \mathrm{mM}$ o-phenanthroline, $10 \mathrm{mM}$ Tris buffer, $\mathrm{pH} 8.0$, containing $0.025 \%$ LDAO and $1 \mathrm{mM}$ EDTA [44]. Approximately $15 \mathrm{mg}$ of RC protein complex embedded in LDAO micelles were used for the NMR experiments.

\subsection{MAS NMR experiments}

NMR experiments were performed with a DMX-200 NMR spectrometer equipped with a double resonance $\mathrm{CP} / \mathrm{MAS}$ probe (Bruker-Biospin, Karlsruhe, Germany). The sample was loaded into a clear 4-mm sapphire rotor and inserted into the MAS probe. The sample was frozen at a low spinning frequency of $600 \mathrm{~Hz}$ to ensure a homogeneous sample distribution [45]. The light and dark spectra were collected using a Hahn echo pulse sequence with the CYCLOPS phase cycle of the $(\pi / 2)$ pulse. The data were collected with TPPM carbon-proton decoupling [38] at a temperature of $223 \mathrm{~K}$ under continuous illumination with white light [46]. The optimum length of the $(\pi / 2)$ carbon pulse, determined on uniformly ${ }^{13} \mathrm{C}$ labelled tyrosine, is $\sim 4.0 \mu \mathrm{s}$ at a strength of $62.5 \mathrm{kHz}$. The rotational frequency for MAS was $8 \mathrm{kHz}$. A total number of 128 scans were collected with a recycle delay of $4 \mathrm{~s}$ and a line broadening of $10 \mathrm{~Hz}$ was applied prior to Fourier transformation.
The ${ }^{13} \mathrm{C}$-MAS NMR spectra were referenced to the ${ }^{13} \mathrm{COOH}$ response of solid tyrosine. $(\mathrm{HCl})$ at $172.1 \mathrm{ppm}$.

\subsection{Lamp set up}

The continuous illumination setup for the MAS NMR experiments comprises a 1000-W xenon arc lamp with collimation optics, a liquid filter and glass filters, a focusing element and a light fiber. Since the emission spectrum of a Xe lamp is similar to sunlight, the full range of radiation from UV to IR is available for illumination. Disturbance of the spinning frequency counting, which operates from a weak light source in the near-IR region, was avoided by a water filter as well as by various Schott filters such as WG320 and KG3. A fiber bundle was used to transfer the radiation from the collimation optics of the lamp to the sample $[2,40]$.

\subsection{1. $2 D{ }^{13} C-{ }^{13} C$ photo-CIDNP DARR MAS NMR under continuous illumination}

All two-dimensional (2D) ${ }^{13} \mathrm{C}-{ }^{13} \mathrm{C}$ photo-CIDNP DARR MAS NMR experiments were recorded using a DMX-200 (4.7 T) Bruker console (Bruker-Biospin, Rheinstetten, Germany). The sample has been frozen in the dark. Samples were kept spinning at $8 \mathrm{kHz}$ at a temperature of $223 \mathrm{~K}$ under continuous illumination with white light [40]. The DARR pulse program was modified to incorporate the excitation with light (Fig. 3B). For DARR experiments, all spectra were collected with $200 t_{1}$ increments and 64 scans, with a recycle delay of $4 \mathrm{~s}$. A spin diffusion mixing time of $1 \mathrm{~s}$ was used.

\subsection{Laser setup}

Using 1064-nm flashes of a Nd:YAG laser (SpectraPhysics Quanta-Ray INDI 40-10, Irvine CA, USA), and frequency-doubling with a second harmonic generator, 532-nm laser flashes were generated with pulse lengths of 6-8 ns and an energy between 20 to $270 \mathrm{~mJ}$. The laser was operating with repetition frequencies between 1 and $4 \mathrm{~Hz}$. Time-resolved photo-CIDNP MAS NMR data were acquired with NMR detection immediately after light excitation, using a presaturation pulse sequence to erase the polarization and coherence from previous scans as described in Daviso et al. [41].

\subsection{1. $2 D{ }^{13} \mathrm{C}-{ }^{13} \mathrm{C}$ photo-CIDNP laser DARR MAS NMR experiments}

The $2 \mathrm{D}{ }^{13} \mathrm{C}-{ }^{13} \mathrm{C}$ photo-CIDNP laser-flash DARR MAS NMR experiment was recorded with the sample spinning at $8 \mathrm{kHz}$ at a temperature of $223 \mathrm{~K}$ using a DMX-200 (4.7 T) Bruker console. The sample was frozen in the dark. The pulse program was modified to incorporate the triggering of the laser pulse CIDNP excitation (Fig. 3C). For laser-flash DARR experiments, all spectra were collected with $100 t_{1}$ increments in 3024 scans, with a recycle delay of $4 \mathrm{~s}$. A spin diffusion mixing time of $1 \mathrm{~s}$ was used.

\subsection{Data processing}

All spectra were processed using the TopSpin (version 2.1) software package (Bruker-Biospin, Karlsruhe, Germany). The cosine square (Qsine with SSB =2) window function was applied along with zero-filling to 1024 data points in both the $t_{1}$ and the $t_{2}$ dimensions.

\subsection{Calculation hyperfine interactions}

DFT Calculations of the Radical Cation of the P. DFT computations of hyperfine coupling tensors were performed with the Amsterdam density functional program (ADF) 2002.1 package (SCM NV), using the triple-Zeta basis set with polarization 

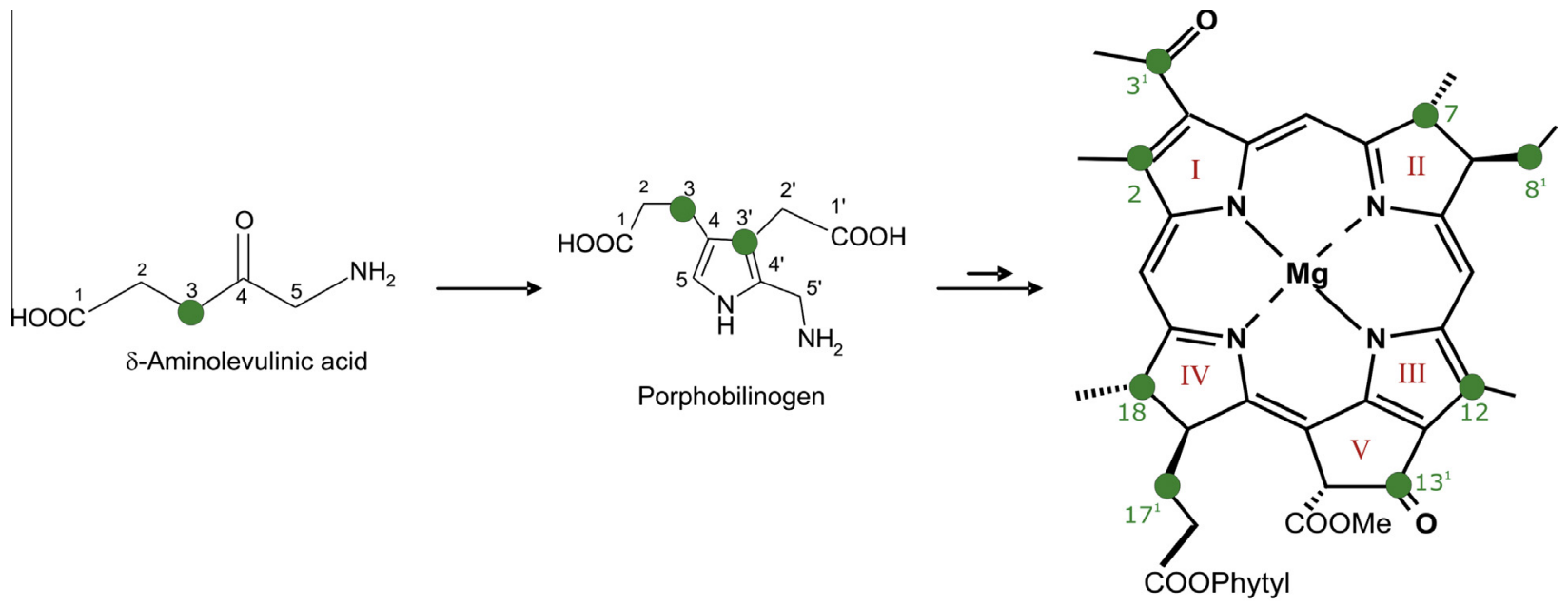

3-ALA-labeled bacteriochlorophyll

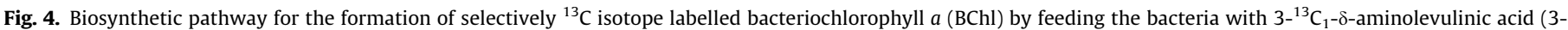
ALA).

functions on all atoms (TZP) all-electron basis set for all atoms as described in Prakash et al. [5] and Daviso et al. [25].

Geometries of ground state molecules were taken from the crystal structure in the charge-neutral state [Protein Data Bank (PDB) ID code 1AIJ] Stowell et al. [47] and subjected to geometry optimization within ADF in the cation radical state.

\section{Results and discussion}

\section{1. $1 D{ }^{13}$ C MAS NMR experiments}

Fig. 5 shows the one-dimensional ${ }^{13} \mathrm{C}$ MAS NMR spectra of 3-ALA labelled RCs of $R$. sphaeroides WT. The data were obtained in a magnetic field of $4.7 \mathrm{~T}$ (i.e., $200 \mathrm{MHz}{ }^{1} \mathrm{H}$ frequency) and at a temperature of $223 \mathrm{~K}$ with a MAS frequency of $8 \mathrm{kHz}$. Fig. 6 provides a detailed view on the carboxylic and aliphatic regions.
Spectrum A in Fig. 5 was collected in the dark, and no signals are observed. Upon CI with white light, strong polarization is established (Spectrum B in Figs. 5 and 6). All signals are light-induced and emissive (negative). The sign of the signal has been explained by the dominance of the TSM over the DD mechanism [4]. The two strongest signals at 119.4 and $134.2 \mathrm{ppm}$ have been assigned to the C-12 and C-2 carbons, respectively. The strong solid-state photoCIDNP effect confirms that these aromatic carbons obtain direct strong enhancement from the electron spin that is delocalized over aromatic system of the special pair [2]. All other signals are considerably weaker. At least four signals can be clearly distinguished in the carbonyl region. The carbonyl carbon atoms are labelled and may gain intensity from the nearby $\mathrm{C}-2$ and $\mathrm{C}-12$ that are strongly polarized. The intensity of the signals in the aliphatic region between 0 and $60 \mathrm{ppm}$ is less than for the signals in the aromatic region. Since for unlabelled RCs under continuous illumination, virtually no photo-CIDNP arises at aliphatic carbons [4], the observed

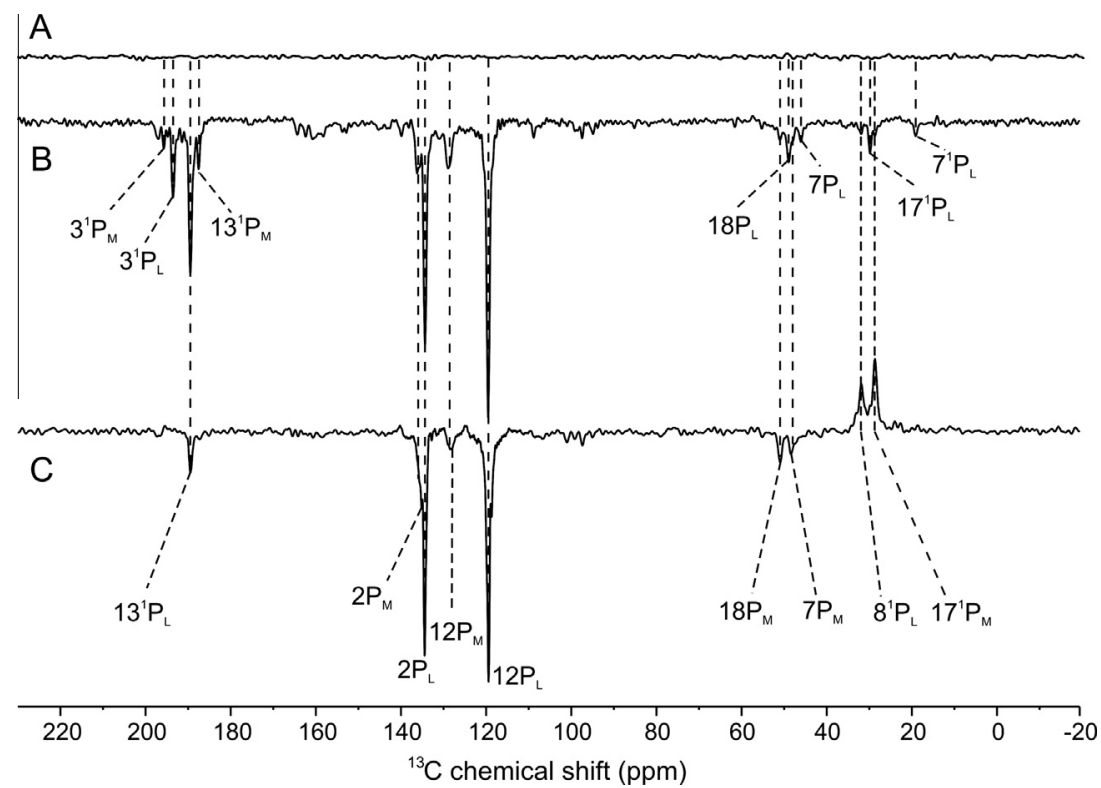

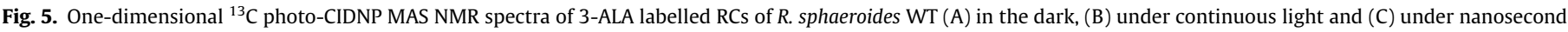
laser-flash with delay $\Delta=0 \mathrm{~ms}$. 


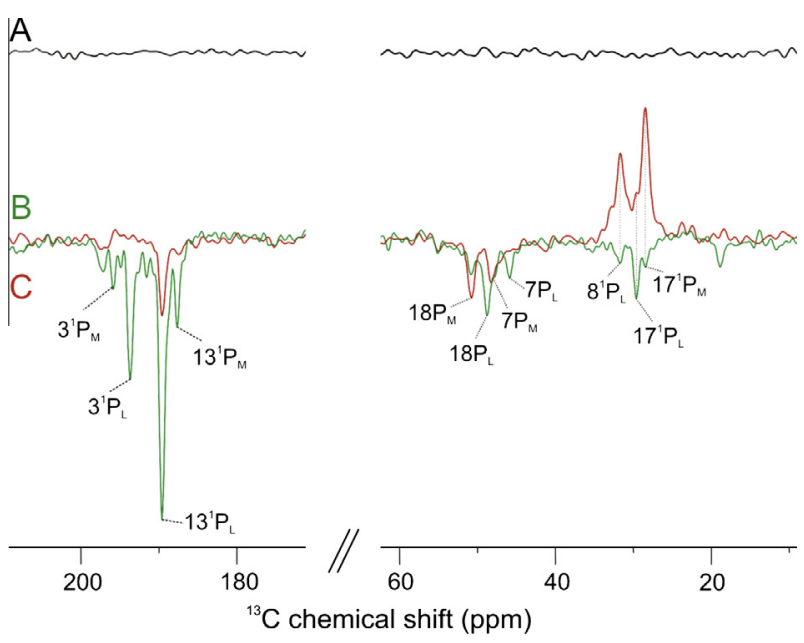

Fig. 6. Carbonyl and aliphatic regions of one-dimensional ${ }^{13} \mathrm{C}$ photo-CIDNP MAS NMR spectra of 3-ALA labelled RCs of $R$. sphaeroides WT (A) in the dark, (B) under continuous light and $(C)$ under nanosecond laser-flash with delay $\Delta=0 \mathrm{~ms}$.

intensity gain in the 1D experiments is attributed to transfer from strongly polarized aromatic carbons by ${ }^{13} \mathrm{C}$ spin diffusion to the aliphatic periphery.

In Spectrum C in Figs. 5 and 6, photo-CIDNP is induced by laser flashes, immediately followed by NMR detection. Compared to the continuous illumination experiment, the signals are both absorptive and emissive since the phase signs of the signals in this spectrum are determined by Kaptein's rule [26]. The occurrence of both emissive and enhanced absorptive lines makes signal assignment easier by referring to calculated local electron spin densities (Tables 2a-2c).

\section{2D ${ }^{13} \mathrm{C}-{ }^{13} \mathrm{C}$ photo-CIDNP DARR MAS NMR spectra}

In Fig. 7, the $2 \mathrm{D}{ }^{13} \mathrm{C}-{ }^{13} \mathrm{C}$ photo-CIDNP DARR MAS NMR spectra of 3-ALA labelled RCs of $R$. sphaeroides WT are shown which have been obtained under $\mathrm{CI}$ with white light (A) and under flash-laser excitation (B). In both cases, the spin-diffusion mixing time was $1 \mathrm{~s}$. In addition to the diagonal peaks, several cross peaks are observed. Many carbons of the BChl cofactors of the special pair have been already assigned in previous studies on unlabelled [4,5], 4-ALA [25,42] and 5-ALA [48] labelled WT RCs. While the 4- and 5-ALA label patterns allow to study the aromatic carbons of the BChl and BPhe macrocycles, 3-ALA labelling leads to isotope enrichment of the more peripheral carbon positions (Figs. 1 and 4). This label pattern is particularly suitable for observation of spin diffusion since it allows for sufficient chemical-shift dispersion for convenient separation of signals.
Table 2a

IUPAC numbers of the $P_{\mathrm{M}}$ and their respective calculated values of $a_{\text {iso }}$ and $\Delta A$.

\begin{tabular}{rcc}
\hline Carbon's position in $P_{\mathrm{M}}$ & $a_{\text {iso }}(\mathrm{MHz})$ & $\Delta A(\mathrm{MHz})$ \\
\hline 1 & 1.7731 & 9.5919 \\
2 & 1.6897 & 9.3966 \\
3 & -1.9722 & 0.2773 \\
4 & 3.605 & 11.0715 \\
5 & -3.4790 & 1.7564 \\
6 & 4.8412 & 13.4397 \\
7 & -1.3268 & 0.5416 \\
8 & -1.6062 & 0.3848 \\
9 & 4.8848 & 14.6508 \\
10 & -2.8147 & 0.5566 \\
11 & 1.6644 & 10.5769 \\
12 & 2.1074 & 12.0685 \\
13 & -2.0735 & 0.614 \\
14 & 3.5553 & 10.843 \\
15 & -3.8828 & 1.9422 \\
16 & 5.8395 & 15.6412 \\
17 & -1.6268 & 0.3458 \\
18 & -1.4505 & 0.2939 \\
19 & 4.78 & 13.977 \\
20 & -3.0592 & 1.2769 \\
$2^{1}$ & -1.1780 & 0.4416 \\
$3^{1}$ & -0.0643 & 0.1084 \\
$3^{2}$ & -0.0091 & 0.1505 \\
$7^{1}$ & 5.433 & 0.9927 \\
$8^{1}$ & 5.426 & 1.1174 \\
$8^{2}$ & 0.1023 & 0.279 \\
$12^{1}$ & -1.5083 & 0.3457 \\
$13^{1}$ & -0.4788 & 0.3528 \\
$13^{2}$ & 0.0875 & 0.1821 \\
$13^{3}$ & -0.8577 & 0.2021 \\
$13^{4}$ & 0.0472 & 0.1121 \\
$17^{1}$ & 4.5759 & 0.3259 \\
$17^{2}$ & -0.0690 & 0.0973 \\
$17^{3}$ & 0.0443 & \\
$18^{1}$ & 5.2977 & \\
& & \\
& & \\
\hline
\end{tabular}

\subsection{Continuous illumination $2 D^{13} \mathrm{C}-{ }^{13} \mathrm{C}$ photo-CIDNP DARR MAS NMR spectrum}

In photo-CIDNP MAS NMR spectra on RCs of $R$. sphaeroides WT, signals from three cofactors are enhanced, the two BChl cofactors of the special pair and the primary electron acceptor BPhe in the active A branch [42]. In the DARR spectrum A of Fig. 7, obtained under $\mathrm{CI}$, however, two networks are observed only, which can be conveniently assigned to the two BChl cofactors of the special pair. The more extended network is labelled in blue and is assigned to cofactor $\mathrm{P}_{\mathrm{L}}$ of the special pair carrying higher electron spin density $[4,49]$. It represents a complete set of correlation signals for eight carbon nuclei, including the responses from the $\mathrm{C}-2$ and the C-12 with the strongest intensities. From this network, a complete set of eight chemical shift assignments have been obtained (Table 1) matching to known resonances of $P_{L}$. A second network, labelled in red, is from a spin system comprising five ${ }^{13} \mathrm{C}$ nuclei,

Table 1

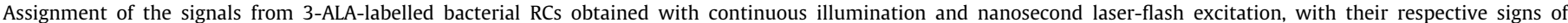
polarization. For the continuous illumination experiment all the polarization is emissive. ( $E=$ emissive; $A=a b s o r p t i v e)$.

\begin{tabular}{|c|c|c|c|c|c|}
\hline Carbon's position in BChl $a$ & $P_{\mathrm{L}}$ & Sign in laser expt & $P_{\mathrm{M}}$ & Sign in laser expt & BPhe \\
\hline 2 & 134.3 & $\mathrm{E}$ & 136.2 & $\mathrm{E}$ & \\
\hline $3^{1}$ & 193.5 & - & 195.6 & - & \\
\hline 7 & 46.1 & - & 48.3 & $\mathrm{E}$ & \\
\hline $8^{1}$ & 32.1 & A & & & \\
\hline 12 & 119.5 & $E$ & 128.8 & $\mathrm{E}$ & 126.9 \\
\hline $13^{1}$ & 189.4 & $\mathrm{E}$ & 187.5 & - & \\
\hline $17^{1}$ & 29.9 & A & 28.4 & A & \\
\hline 18 & 49.1 & - & 50.9 & $\mathrm{E}$ & \\
\hline $7^{1}$ & 19.1 & - & & - & \\
\hline
\end{tabular}


Table 2b

IUPAC numbers of the $P_{\mathrm{L}}$ and their respective calculated values of $a_{\text {iso }}$ and $\Delta \underline{A}$.

\begin{tabular}{|c|c|c|}
\hline Carbon's position in $P_{\mathrm{L}}$ & $a_{\text {iso }}(\mathrm{MHz})$ & $\Delta A(\mathrm{MHz})$ \\
\hline 1 & 2.8829 & 14.3282 \\
\hline 2 & 2.158 & 13.113 \\
\hline 3 & -2.3671 & 1.7295 \\
\hline 4 & 5.2484 & 15.8799 \\
\hline 5 & -4.7583 & 2.269 \\
\hline 6 & 7.0186 & 19.1645 \\
\hline 7 & -1.9269 & 0.6833 \\
\hline 8 & -2.2206 & 0.3978 \\
\hline 9 & 6.8934 & 20.1713 \\
\hline 10 & -4.5494 & 1.6325 \\
\hline 11 & 2.6737 & 15.545 \\
\hline 12 & 2.806 & 16.5525 \\
\hline 13 & -2.6225 & 1.3671 \\
\hline 14 & 4.7728 & 14.7333 \\
\hline 15 & -5.3101 & 2.2941 \\
\hline 16 & 9.2318 & 24.4369 \\
\hline 17 & -2.5917 & 0.5872 \\
\hline 18 & -2.0785 & 0.6113 \\
\hline 19 & 8.0712 & 20.8362 \\
\hline 20 & -4.4822 & 1.9127 \\
\hline $2^{1}$ & -1.6535 & 0.307 \\
\hline $3^{1}$ & -0.5444 & 0.3567 \\
\hline $3^{2}$ & -0.1322 & 0.1365 \\
\hline $7^{1}$ & 6.8887 & 1.3155 \\
\hline $8^{1}$ & 7.4891 & 1.5265 \\
\hline $8^{2}$ & 0.1731 & 0.4405 \\
\hline $12^{1}$ & -2.0383 & 0.5073 \\
\hline $13^{1}$ & -0.7157 & 0.4978 \\
\hline $13^{2}$ & 0.2166 & 0.2119 \\
\hline $13^{3}$ & -1.2329 & 0.1742 \\
\hline $13^{4}$ & -0.0330 & 0.0622 \\
\hline $17^{1}$ & 8.7024 & 1.7612 \\
\hline $17^{2}$ & 0.0819 & 0.417 \\
\hline $17^{3}$ & -0.0411 & 0.115 \\
\hline $18^{1}$ & 8.8269 & 1.5838 \\
\hline
\end{tabular}

and is assigned to cofactor $\mathrm{P}_{\mathrm{M}}$. As observed previously, most of the signals assigned to $\mathrm{P}_{\mathrm{L}}$ are shielded compared to the signals of $\mathrm{P}_{\mathrm{M}}$ $[4,27,42,48]$. In particular the difference between the chemical shifts of the C-12 atoms of $9.0 \mathrm{ppm}$ is remarkable and demonstrates the significant ground-state asymmetry within the dimer [42]. Hence, 2D CI experiments allow for full analysis of the electronic ground state

\subsection{Time-resolved $2 D^{13} C_{-}{ }^{13} C$ photo-CIDNP DARR MAS NMR spectrum}

Fig. 7B shows the two-dimensional ${ }^{13} \mathrm{C}-{ }^{13} \mathrm{C}$ photo-CIDNP laserflash DARR MAS NMR spectra of 3-ALA labelled RCs of $R$. sphaeroides WT collected at $223 \mathrm{~K}$ with a delay time $(\Delta)$ between the laser excitation pulse and NMR detection pulse of $0 \mu$ s and a spin-diffusion mixing time of $1 \mathrm{~s}$ at a MAS frequency of $8 \mathrm{kHz}$. In timeresolved experiments on RCs of $R$. sphaeroides WT with virtually no delay time $\Delta$, the light-induced signals are mainly due to the transient nuclear polarization as explained above (Fig. 2) [25]. Hence, the laser-flash ${ }^{13} \mathrm{C}-{ }^{13} \mathrm{C}$ correlation experiment provides both a view on the electronic structure as well as chemical shift assignments of the labelled atoms in the special pair.

In the time-resolved two-dimensional spectrum, mainly peaks from $P_{L}$ appear and only a few from $P_{M}$ (Table 1 ). In the one-dimensional dataset collected with laser-flash excitation, the signals in the aliphatic region are relatively strong compared to the $\mathrm{CI}$ experiment (Fig. 6, right part) and the signals from $C-17^{1} \mathrm{P}_{\mathrm{M}}, \mathrm{C}-17^{1} \mathrm{P}_{\mathrm{L}}$ and $\mathrm{C}-8^{1} \mathrm{P}_{\mathrm{L}}$ are absorptive. In Fig. 7 , selected regions of $2 \mathrm{D}$ spectra are displayed. Since Fig. 7A is obtained with $\mathrm{CI}$, all diagonal peaks are emissive, which leads to completely emissive sets of cross peaks. The cross peak intensities confirm that the signal strength
Table 2c

IUPAC numbers of the $\Phi_{\mathrm{A}}$ and their respective calculated values of $a_{\text {iso }}$ and $\Delta A$.

\begin{tabular}{|c|c|c|}
\hline Carbon's position in $\Phi_{\mathrm{A}}$ & $a_{\text {iso }}(\mathrm{MHz})$ & $\Delta A(\mathrm{MHz})$ \\
\hline 1 & -4.0898 & 7.5168 \\
\hline 2 & 8.4399 & 33.5792 \\
\hline 3 & -0.5842 & 12.9589 \\
\hline 4 & -3.6908 & 0.8122 \\
\hline 5 & 6.2948 & 22.1958 \\
\hline 6 & -6.4808 & 2.868 \\
\hline 7 & 0.1378 & 0.3274 \\
\hline 8 & 0.1277 & 0.2887 \\
\hline 9 & -5.8094 & 1.9962 \\
\hline 10 & 5.1275 & 19.8083 \\
\hline 11 & -3.7464 & 5.2241 \\
\hline 12 & 7.4997 & 28.5875 \\
\hline 13 & -1.9219 & 8.8494 \\
\hline 14 & -4.0378 & 0.9188 \\
\hline 15 & 8.0234 & 25.5863 \\
\hline 16 & -7.9137 & 3.6307 \\
\hline 17 & 0.2288 & 0.3256 \\
\hline 18 & -0.2166 & 0.348 \\
\hline 19 & 6.2948 & 22.1958 \\
\hline 20 & 6.1744 & 24.4854 \\
\hline $2^{1}$ & -4.3718 & 0.6735 \\
\hline $3^{1}$ & 1.4387 & 10.9881 \\
\hline $3^{2}$ & -1.8654 & 0.2722 \\
\hline $7^{1}$ & -0.3147 & 0.3033 \\
\hline $8^{1}$ & -0.5471 & 0.2329 \\
\hline $8^{2}$ & 0.3963 & 0.2511 \\
\hline $12^{1}$ & -3.6127 & 0.4828 \\
\hline $13^{1}$ & 1.5118 & 10.8316 \\
\hline $13^{2}$ & -4.0240 & 0.203 \\
\hline $13^{3}$ & 0.4748 & 0.4701 \\
\hline $13^{4}$ & -0.0560 & 0.1806 \\
\hline $17^{1}$ & -0.6292 & 0.2518 \\
\hline $17^{2}$ & 0.1759 & 0.1519 \\
\hline $17^{3}$ & -0.0083 & 0.0801 \\
\hline $18^{1}$ & 0.8611 & 0.3309 \\
\hline
\end{tabular}

is transferred along the $f_{2}$ dimension. For example, the cross peak, $\mathrm{C}-12 \mathrm{P}_{\mathrm{L}} / \mathrm{C}-7^{1} \mathrm{P}_{\mathrm{L}}$ is very pronounced, as the corresponding diagonal peak in the $f_{2}$ direction is also strong. In Fig. 7B, laser flashes were used as source of the photo-CIDNP enhancement and both absorptive and emissive signals occur. The cross peaks in the aliphatic region, for example, $\mathrm{C}-18 \mathrm{P}_{\mathrm{M}} / \mathrm{C}-17^{1} \mathrm{P}_{\mathrm{M}}$ show the same sign as the diagonal peaks along the $f_{2}$ dimension (see dashed arrows).

Compared to the continuous illumination experiment, in timeresolved experiments, though the signs of the peak directly follow the Kaptein's rules. It should be noted that different orientations contribute differently to the RPM because of the anisotropy of the $\mathrm{g}$ factor difference. Hence, if hyperfine anisotropy is much larger than the isotropic hyperfine coupling, the orientation average of RPM is not necessarily proportional to $a_{\text {iso }}$. For the nuclei where the isotropic hyperfine coupling dominates the anisotropic coupling $\left(\mathrm{C}-177^{1} \mathrm{P}_{\mathrm{M}}, \mathrm{C}-8{ }^{1} \mathrm{P}_{\mathrm{L}}, \mathrm{C}-7 \mathrm{P}_{\mathrm{M}}, \mathrm{C}-18 \mathrm{P}_{\mathrm{M}}, \mathrm{C}-3^{1} \mathrm{P}_{\mathrm{L}}\right)$, so that the sign of the hyperfine coupling is the same at all orientations, the sign of the signal agrees with the sign of $a_{\text {iso }}$. For strong hyperfine anisotropy $\left(\mathrm{C}-2 \mathrm{P}_{\mathrm{L}}, \mathrm{C}-12 \mathrm{P}_{\mathrm{L}}, \mathrm{C}-2 \mathrm{P}_{\mathrm{M}}, \mathrm{C}-12 \mathrm{P}_{\mathrm{M}}\right)$ negative signals are observed even if $a_{\text {iso }}$ is positive, which may result from contributions by the TSM/DD solid-state CIDNP mechanisms or from weighted orientation averaging of the RPM contributions. For dominating hyperfine anisotropy, orientations with negative as well as positive sign of the hyperfine coupling exist and the signal contribution also depends on the anisotropic $g$ value difference between donor and acceptor [25,27]. Therefore fewer diagonal peaks are observed for the same mixing time of $1 \mathrm{~s}$. Hence, the enhancement mechanism in the 2D laser-DARR acts as a filter for correlation experiments. The higher selectivity also allows for clarifying the assignments for $\mathrm{C}-7 \mathrm{P}_{\mathrm{M}}, \mathrm{C}-7 \mathrm{P}_{\mathrm{M}}, \mathrm{C}-8 \mathrm{P}_{\mathrm{L}}$ and $\mathrm{C}-18 \mathrm{P}_{\mathrm{M}}$. (Table 1 ). 


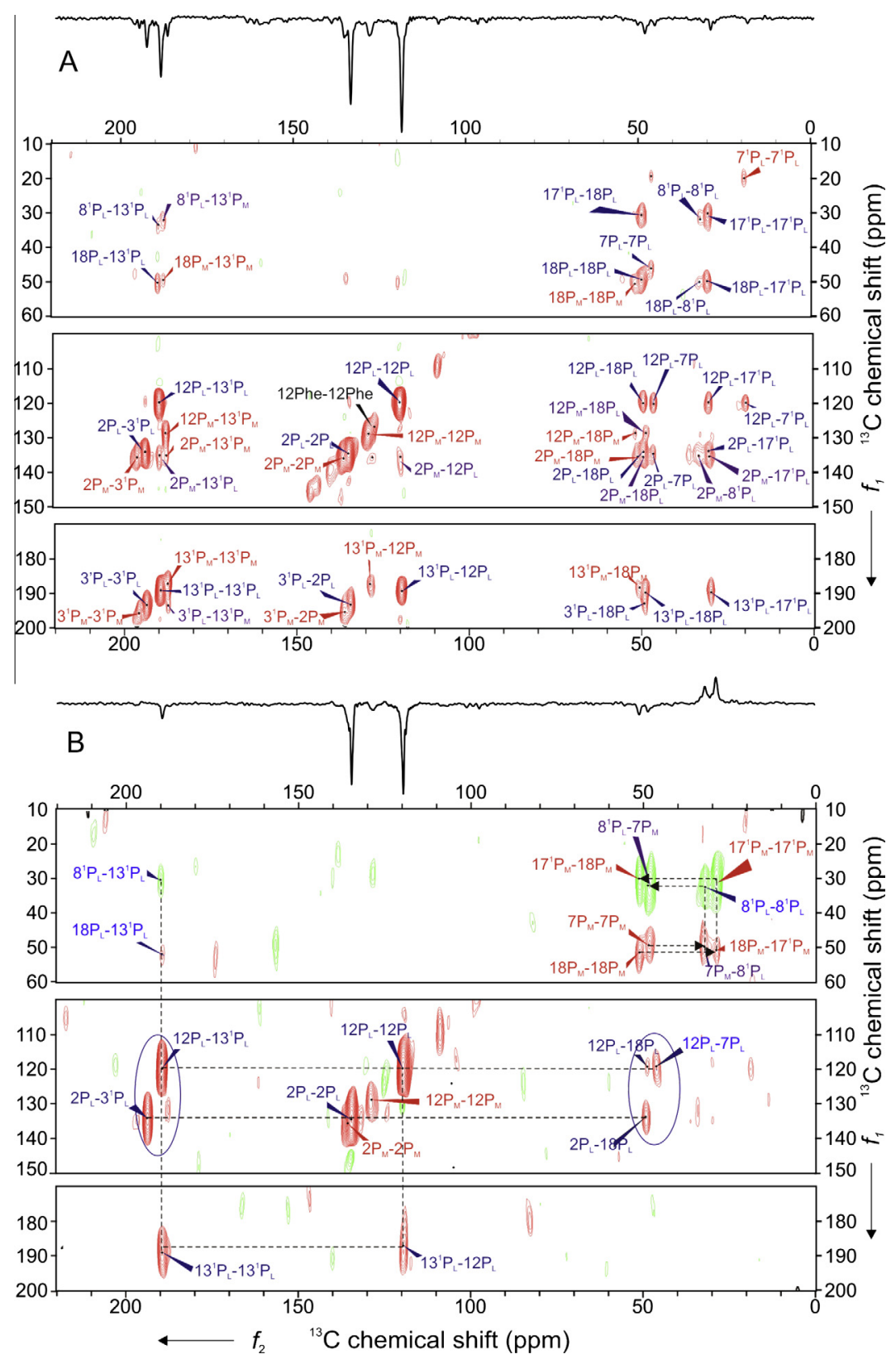

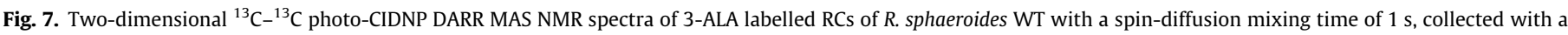

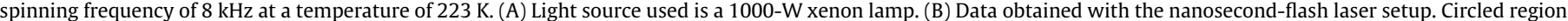

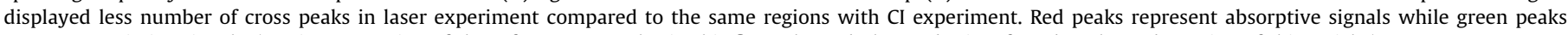
represent emissive signals. (For interpretation of the references to color in this figure legend, the reader is referred to the web version of this article.)

\section{Conclusions}

The complex signal pattern observed in the photo-CIDNP MAS 2D NMR experiment spectrum under $\mathrm{CI}$ has been filtered and edited in the time-resolved experiment by the values and signs of isotropic $\left(a_{\text {iso }}\right)$ and anisotropic $(\Delta A)$ hyperfine interaction. This method allows to refine assignments and to validate calculated local electron spin densities. In addition, local electron spin densities can be directly mapped from radical pairs, due to the strong photo-CIDNP enhancement, even when they originate from membrane bound proteins.

\section{References}

[1] G. Jeschke, J. Matysik, A reassessment of the origin of photochemically induced dynamic nuclear polarization effects in solids, Chem. Phys. 294 (2003) 239-255.

[2] E. Daviso, G. Jeschke, J. Matysik, Photochemically induced dynamic nuclear polarization (Photo-CIDNP) magic-angle spinning NMR, in: T. Aartsma, J.
Matysik (Eds.), Biophysical Techniques in Photosynthesis II, Springer, Dordrecht, 2008, pp. 385-399.

[3] M.G. Zysmilich, A. McDermott, Photochemically induced dynamic nuclearpolarization in the solid-state ${ }^{15} \mathrm{~N}$ spectra of reaction centers from photosynthetic bacteria Rhodobacter sphaeroides R-26, J. Am. Chem. Soc. 116 (1994) 8362-8363.

[4] S. Prakash, Alia, P. Gast, H.J.M. de Groot, G. Jeschke, J. Matysik, Magnetic field dependence of photo-CIDNP MAS NMR on photosynthetic reaction centers of Rhodobacter sphaeroides WT, J. Am. Chem. Soc. 127 (2005) 14290-14298.

[5] S. Prakash, Alia, P. Gast, H.J.M. de Groot, J. Matysik, G. Jeschke, Photo-CIDNP MAS NMR in intact cells of Rhodobacter sphaeroides R-26: Molecular and atomic resolution at nanomolar concentration, J. Am. Chem. Soc. 128 (2006) 12794-12799.

[6] E. Roy, T. Rohmer, P. Gast, G. Jeschke, A. Alia, J. Matysik, Characterization of the primary radical pair in reaction centers of Heliobacillus mobilis by ${ }^{13} \mathrm{C}$ PhotoCIDNP MAS NMR, Biochemistry 47 (2008) 4629-4635.

[7] S.S. Thamarath, B.E. Bode, S. Prakash, K.B. Sai Sankar Gupta, A. Alia, G. Jeschke, J Matysik, Electron spin density Distribution in the special pair Triplet of Rhodobacter sphaeroides R26 revealed by magnetic field dependence of the solid-state Photo-CIDNP effect, J. Am. Chem. Soc. 134 (2012) 5921-5930. 
[8] A. Diller, A. Alia, P. Gast, G. Jeschke, J. Matysik, ${ }^{13} \mathrm{C}$ Photo-CIDNP MAS NMR on the LH1-RC complex of Rhodopseudomonas acidophila, in: Photosynthesis. Energy from the Sun, 2008, pp. 55-58.

[9] E. Roy, Alia, P. Gast, H. van Gorkom, H.J.M. de Groot, G. Jeschke, J. Matysik Photochemically induced dynamic nuclear polarization in the reaction center of the green sulphur bacterium Chlorobium tepidum observed by ${ }^{13} \mathrm{C}$ MAS NMR, Biochim. Biophys. Acta - Bioenerg. 1767 (2007) 610-615.

[10] J. Matysik, Alia, P. Gast, H.J. van Gorkom, A.J. Hoff, H.J.M. de Groot Photochemically induced nuclear spin polarization in reaction centers of photosystem II observed by ${ }^{13} \mathrm{C}$-solid-state $\mathrm{NMR}$ reveals a strongly asymmetric electronic structure of the P680.+ primary donor chlorophyll, Proc. Natl. Acad. Sci. U. S. A 97 (2000) 9865-9870.

[11] A. Alia, E. Roy, P. Gast, H.J. van Gorkom, H.J.M. de Groot, G. Jeschke, J. Matysik, Photochemically induced dynamic nuclear polarization in photosystem I of plants observed by ${ }^{13} \mathrm{C}$ magic-angle spinning NMR, J. Am. Chem. Soc. 126 (2004) 12819-12826.

[12] A. Diller, E. Roy, P. Gast, H.J. van Gorkom, H.J.M. de Groot, C. Glaubitz, G. Jeschke, J. Matysik, A. Alia, ${ }^{15} \mathrm{~N}$ photochemically induced dynamic nuclear polarization magic-angle spinning NMR analysis of the electron donor of photosystem II, Proc. Natl. Acad. Sci. U. S. A. 104 (2007) 12767-12771.

[13] G.J. Janssen, E. Daviso, M. van Son, H.J.M. de Groot, A. Alia, J. Matysik, Observation of the solid-state photo-CIDNP effect in entire cells of cyanobacteria Synechocystis, Photosynth. Res. 104 (2010) 275-282.

[14] J. Matysik, A. Diller, E. Roy, A. Alia, The solid-state photo-CIDNP effect, Photosynth. Res. 102 (2009) 427-435.

[15] S. Thamarath Surendran, J. Heberle, P.J. Hore, T. Kottke, J. Matysik, Solid-state photo-CIDNP effect observed in phototropin LOV1-C57S by ${ }^{13} \mathrm{C}$ magic-angle spinning NMR spectroscopy, J. Am. Chem. Soc. 132 (2010) 15542-15543.

[16] A.J. Hoff, J. Deisenhofer, Photophysics of photosynthesis. Structure and spectroscopy of reaction centers of purple bacteria, Phys. Rep. - Rev. Sect. Phys. Lett. 287 (1997) 2-247.

[17] R.E. Blankenship, G.T. Babcock, J.T. Warden, K. Sauer, Observation of a new EPR transient in chloroplasts that may reflect electron-donor to photosystem II at room-temperature, FEBS Lett. 51 (1975) 287-293.

[18] A.J. Hoff, H. Rademaker, R. Vangrondelle, L.N.M. Duysens, Magnetic-field dependence of yield of triplet-state in reaction centers of photosynthetic bacteria, Biochim. Biophys. Acta 460 (1977) 547-554.

[19] G.L. Closs, L.E. Closs, Induced dynamic nuclear spin polarization in reactions of photochemically and thermally generated triplet diphenylmethylene, J. Am. Chem. Soc. 91 (1969) 4549.

[20] R. Kaptein, J.L. Oosterhoff, Chemically induced dynamic nuclear polarization II: (Relation with anomalous ESR spectra), Chem. Phys. Lett. 4 (1969) 195.

[21] G. Jeschke, Electron-electron-nuclear three-spin mixing in spin-correlated radical pairs, J. Chem. Phys. 106 (1997) 10072-10086.

[22] G. Jeschke, A new mechanism for chemically induced dynamic nuclear polarization in the solid state, J. Am. Chem. Soc. 120 (1998) 4425-4429.

[23] T. Polenova, A.E. McDermott, A coherent mixing mechanism explains the photoinduced nuclear polarization in photosynthetic reaction centers, J. Phys. Chem. B 103 (1999) 535-548.

[24] A. Diller, S. Prakash, A. Alia, P. Gast, J. Matysik, G. Jeschke, Signals in solid-state photochemically induced dynamic nuclear polarization recover faster than signals obtained with the longitudinal relaxation time, J. Phys. Chem. B 111 (2007) 10606-10614.

[25] E. Daviso, A. Alia, S. Prakash, A. Diller, P. Gast, J. Lugtenburg, J. Matysik, G. Jeschke, Electron-nuclear spin dynamics in a bacterial photosynthetic reaction center, J. Phys. Chem. C 113 (2009) 10269-10278.

[26] R. Kaptein, Simple rules for chemically induced dynamic nuclear polarization, Chem. Commun. 14 (1971) 732-733.

[27] E. Daviso, S. Prakash, A. Alia, P. Gast, J. Neugebauer, G. Jeschke, J. Matysik, The electronic structure of the primary electron donor of reaction centers of purple bacteria at atomic resolution as observed by photo-CIDNP ${ }^{13} \mathrm{C}$ NMR, Proc. Natl. Acad. Sci. U. S. A. 106 (2009) 22281-22286.

[28] R. Tycko, G. Dabbagh, Measurement of nuclear magnetic dipole-dipole couplings in magic angle spinning NMR, Chem. Phys. Lett. 173 (1990) 461-465.

[29] A.E. Bennett, R.G. Griffin, J.H. Ok, S. Vega, Chemical shift correlation spectroscopy in rotating solids: radio frequency-driven dipolar recoupling and longitudinal exchange, J. Chem. Phys. 96 (1992) 8624-8627.
[30] A.E. Bennett, C.M. Rienstra, J.M. Griffiths, W. Zhen, J.P.T. Lansbury, R.G. Griffin, Homonuclear radio frequency-driven recoupling in rotating solids, J. Chem. Phys. 108 (1998) 9463-9479.

[31] Y.K. Lee, N.D. Kurur, M. Helmle, O.G. Johannessen, N.C. Nielsen, M.H. Levitt, Efficient dipolar recoupling in the NMR of rotating solids - a sevenfold symmetrical radiofrequency pulse sequence, Chem. Phys. Lett. 242 (1995) 304-309.

[32] N.M. Szeverenyi, M.J. Sullivan, G.E. Maciel, Observation of spin exchange by two-dimensional Fourier-transform C13 cross polarization-magic-angle spinning, J. Magn. Reson. 47 (1982) 462-475.

[33] A. Grommek, B.H. Meier, M. Ernst, Distance information from proton-driven spin diffusion under MAS, Chem. Phys. Lett. 427 (2006) 404-409.

[34] K. Takegoshi, S. Nakamura, T. Terao, ${ }^{13} \mathrm{C}-{ }^{1} \mathrm{H}$ dipolar-assisted rotational resonance in magic angle spinning NMR, Chem. Phys. Lett. 344 (2001) 631637.

[35] N. Nielsen, L. Strassø, A. Nielsen, Dipolar recoupling, in: J.C.C. Chan (Ed.), Solid State NMR, Springer, Berlin Heidelberg, 2012, pp. 1-45.

[36] S. Dusold, A. Sebald, Dipolar recoupling under magic-angle spinning conditions, in: Annual Reports on NMR Spectroscopy, Academic Press, 2000, pp. 185-264.

[37] M. Veshtort, R.G. Griffin, Proton-driven spin diffusion in rotating solids via reversible and irreversible quantum dynamics, J. Chem. Phys. 135 (2011) 134509.

[38] A.E. Bennett, C.M. Rienstra, M. Auger, K.V. Lakshmi, R.G. Griffin, Heteronuclear decoupling in rotating solids, J. Chem. Phys. 103 (1995) 6951-6958.

[39] N.C. Nielsen, F. Creuzet, R.G. Griffin, M.H. Levitt, Enhanced double-quantum nuclear magnetic resonance in spinning solids at rotational resonance, J. Chem. Phys. 96 (1992) 5668.

[40] J. Matysik, Alia, J. Holland, T. Egorova-Zachernyuk, P. Gast, H.J.M. de Groot, Sample illumination and photo-CIDNP in a magic-angle spinning NMR probe, Indian J. Biochem. Biophys. 37 (2000) 418-423.

[41] E. Daviso, A. Diller, A. Alia, J. Matysik, G. Jeschke, Photo-CIDNP MAS NMR beyond the $\mathrm{T}_{1}$ limit by fast cycles of polarization extinction and polarization generation, J. Magn. Reson. 190 (2008) 43-51.

[42] E.A.M. Schulten, J. Matysik, Alia, S. Kiihne, J. Raap, J. Lugtenburg, P. Gast, A.J. Hoff, H.J.M. de Groot, ${ }^{13} \mathrm{C}$ MAS NMR and photo-CIDNP reveal a pronounced asymmetry in the electronic ground state of the special pair of Rhodobacter sphaeroides reaction centers, Biochemistry 41 (2002) 8708-8717.

[43] S. Shochat, T. Arlt, C. Francke, P. Gast, P.I. Vannoort, S.C.M. Otte, H.P.M. Schelvis, S. Schmidt, E. Vijgenboom, J. Vrieze, W. Zinth, A.J. Hoff, Spectroscopic characterization of reaction centers of the (M)Y210W mutant of the photosynthetic bacterium Rhodobacter sphaeroides, Photosynth. Res. 40 (1994) 55-66.

[44] M.Y. Okamura, R.A. Isaacson, G. Feher, Primary acceptor in bacterial photosynthesis - obligatory role of ubiquinone in photoactive reaction centers of Rhodopseudomonas spheroides, Proc. Natl. Acad. Sci. U. S. A. 72 (1975) 3491-3495.

[45] M.R. Fischer, H.J.M. Degroot, J. Raap, C. Winkel, A.J. Hoff, J. Lugtenburg, ${ }^{13} \mathrm{C}$ magic angle spinning NMR-study of the light-induced and temperaturedependent changes in Rhodobacter sphaeroides $\mathrm{R}-26$ reaction centers enriched in $4^{\prime}-{ }^{13} \mathrm{C}$ tyrosine, Biochemistry 31 (1992) $11038-11049$.

[46] J. Matysik, E. Schulten, Alia, P. Gast, J. Raap, J. Lugtenburg, A.J. Hoff, H.J.M. de Groot, Photo-CIDNP 13C magic angle spinning NMR on bacterial reaction centres: exploring the electronic structure of the special pair and its surroundings, Biol. Chem. 382 (2001) 1271-1276.

[47] M.H.B. Stowell, T.M. McPhillips, D.C. Rees, S.M. Soltis, E. Abresch, G. Feher, Light-induced structural changes in photosynthetic reaction center: implications for mechanism of electron-proton transfer, Science 276 (1997) 812-816.

[48] S. Prakash, A. Alia, P. Gast, H.J.M. de Groot, G. Jeschke, J. Matysik, ${ }^{13} \mathrm{C}$ chemical shift map of the active cofactors in photosynthetic reaction centers of Rhodobacter sphaeroides revealed by photo-CIDNP MAS NMR, Biochemistry 46 (2007) 8953-8960.

[49] A. Marchanka, W. Lubitz, M. van Gastel, Spin density distribution of the excited triplet state of bacteriochlorophylls. Pulsed ENDOR and DFT studies, J. Phys. Chem. B 113 (2009) 6917-6927. 